\title{
Inducing chromosome pairing through premature condensation: analysis of wheat interspecific hybrids
}

\author{
Emilie Knight • Emma Greer • Tracie Draeger • \\ Vera Thole $\cdot$ Steve Reader • Peter Shaw • \\ Graham Moore
}

Received: 19 May 2010 / Revised: 13 July 2010 / Accepted: 18 July 2010 /Published online: 31 July 2010

(C) The Author(s) 2010. This article is published with open access at Springerlink.com

\begin{abstract}
At the onset of meiosis, chromosomes first decondense and then condense as the process of recognition and intimate pairing occurs between homologous chromosomes. We show here that okadaic acid, a drug known to induce chromosome condensation, can be introduced into wheat interspecific hybrids prior to meiosis to induce chromosome pairing. This pairing occurs in the presence of the Phl locus, which usually suppresses pairing of related chromosomes and which we show here delays condensation. Thus the timing of chromosome condensation during the onset of meiosis is an important factor in controlling chromosome pairing.
\end{abstract}

Keywords $P h 1$ locus $\cdot$ Chromatin $\cdot$ Meiosis $\cdot$ Prebreeding

\section{Introduction}

Many plant species are polyploid. Despite possessing multiple genomes, only true homologous chromosomes pair during meiosis. This correct pairing involves suppressing pairing between the multiple sets of related chromosomes. Understanding the basis for this pairing suppression has a practical application potentially enabling the effect to

Electronic supplementary material The online version of this article (doi:10.1007/s10142-010-0185-0) contains supplementary material, which is available to authorized users.

E. Knight • E. Greer · T. Draeger · V. Thole · S. Reader • P. Shaw $\cdot$

G. Moore $(\bowtie)$

John Innes Centre,

Colney,

Norwich NR4 7UH, UK

e-mail: graham.moore@bbsrc.ac.uk be switched on and off, thereby enhancing breeding strategies (Able and Langridge 2006). The pairing process involves the telomeres of the chromosomes clustering to form a bouquet at the onset of meiosis. Chromosomes then initiate intimate pairing or synapsis from the telomere regions. Early meiosis is also associated with marked changes in chromosome structure. Chromosome pairing in wheat is controlled by a single major locus, $P h l$ (reviewed by Yousafzai et al. 2010a). Wheat lacking the Phl locus accumulates chromosome rearrangements and eventually becomes infertile (Sanchez-Moran et al. 2001). Sexual hybridisation between wheat and a wild relative generally produces an interspecific hybrid containing a haploid set of only related chromosomes, in which there is no chromosome pairing due to the presence of the $P h l$ locus (Riley et al. 1959). Molecular analysis of the $P h l$ locus has defined it to a region on chromosome $5 \mathrm{~B}$ containing a cluster of seven cdc2-like genes (Griffiths et al. 2006; Al-Kaff et al. 2008), with homology to $c d k 2$ also required for correct pairing in mammals (Yousafzai et al. 2010b; Viera et al. 2009). We have exploited our understanding of the Phl locus to demonstrate here that regulation of chromosome condensation and pairing between related chromosomes are linked.

\section{Materials and methods}

Plant material

The tillers used for this study came from hexaploid wheat, Triticum aestivum cv. Beaver, crosses between hexaploid wheat cv. Hobbit sib and rye (Secale cereale) cv. Petkus and also between T. aestivum cv. Chinese Spring and rye cv. Petkus either carrying or lacking the $P h l$ locus. 
Fig. 1 TaASY1 localisation (red) at early meiosis in wildtype (a, c, e, g) and $P h 1$ mutant wheat-rye $F_{1}$ wheat (b, d,

f, h). Meiocytes at pre-telomere bouquet $(\mathbf{a}, \mathbf{b})$, early bouquet (c, d), late bouquet $(\mathbf{e}, \mathbf{f})$, as bouquet disperses $(\mathbf{g}, \mathbf{h})$. Scale bars $5 \mu \mathrm{m}$

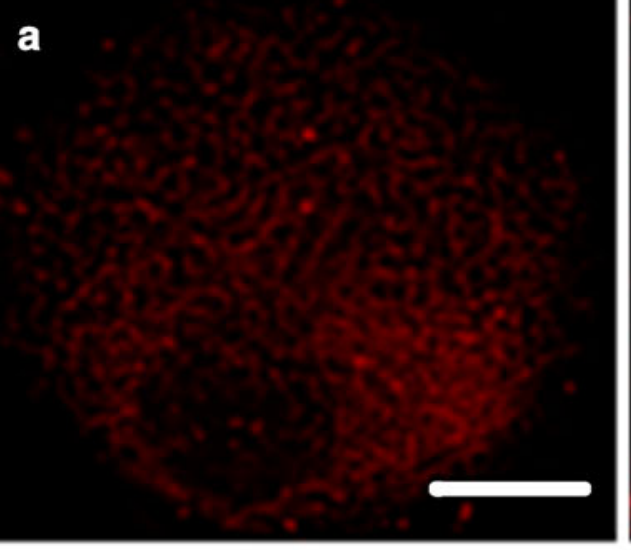

b
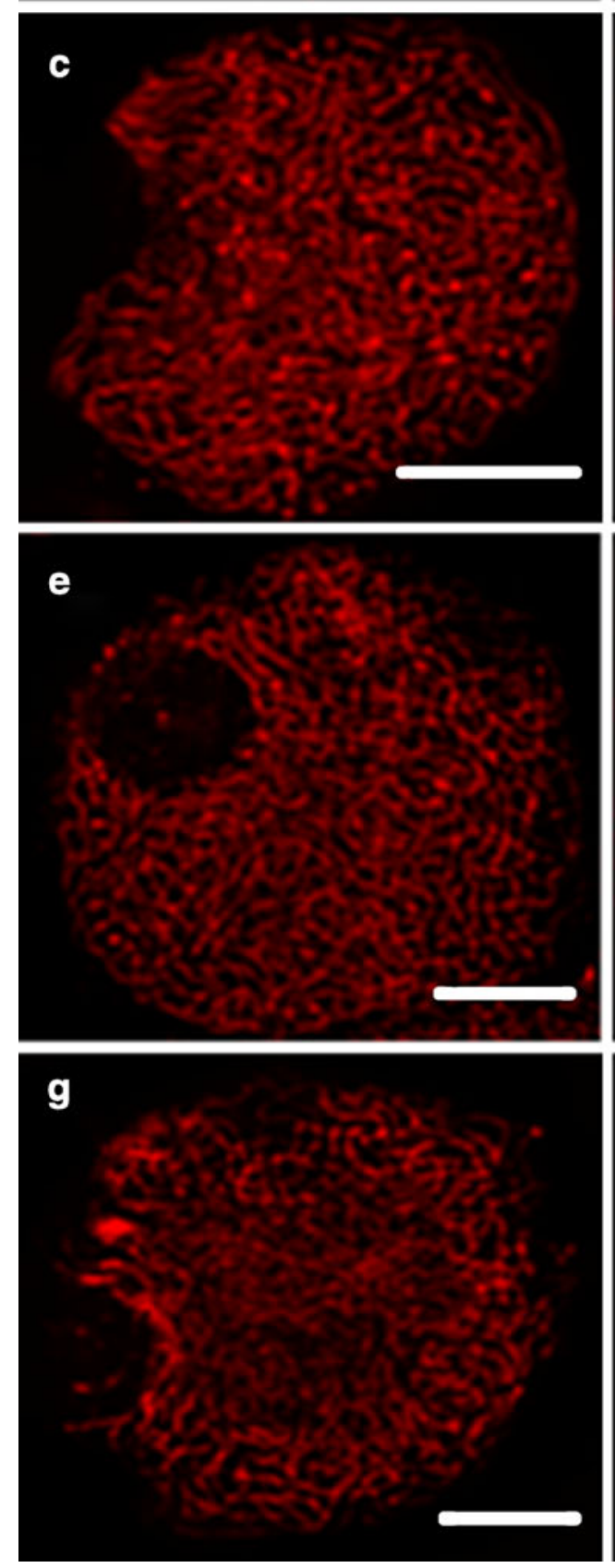

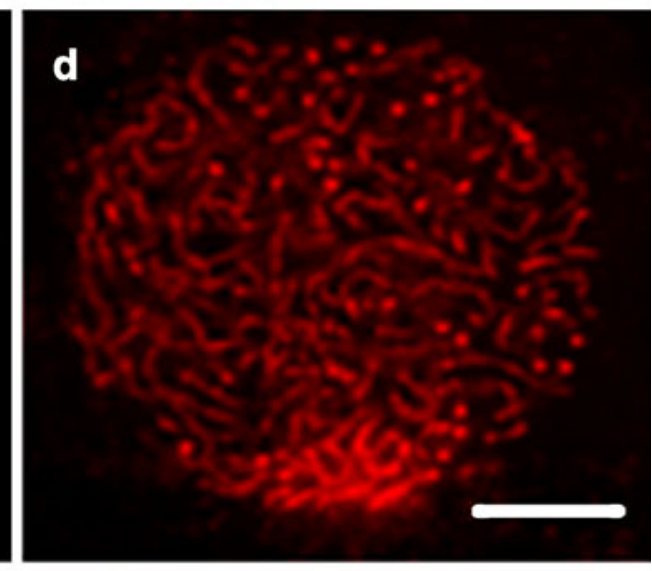

f

h 
Sectioning, fluorescence in situ hybridisation, microscopy and image processing

The tissue sectioning, specimen preparation, in situ hybridisation, fluorescence microscopy and image processing have all been described previously (Prieto et al. 2007). Axial element lengths were determined using ImageJ by counting the total pixels of skeletonised Asy1-labelled elements. The TaAsyl antibody was kindly provided by Dr. Jason Able and the fluorescence immuno-localisation was performed as described in Boden et al., using the rabbit anti-TaAsy1 antibody and AlexFluor 568 conjugated donkey anti-rabbit antibody (Boden et al. 2009).

Antisense oligodeoxynucleotide inhibition and okadaic acid treatment using a detached tiller method

Tillers containing an immature pre-meiotic spike were detached immediately after an 8-h dark period and transferred immediately to a solution of $100 \mathrm{mM}$ sucrose and antisense oligodeoxynucleotides (ODNs; combination of either four sense or antisense ODNs at $10 \mu \mathrm{M}$ each). The cut tillers in individual tubes were then placed in the dark for 3 to 4 days (until the spike reached meiosis), after which the dark cover was removed for 2-3 $\mathrm{h}$ and spikes were then dissected out. Anthers with pollen mother cells in meiosis were removed and fixed in one part glacial acetic acid to three parts ethanol and subsequently stained. Excised Feulgen stained anthers were squashed in aceto-carmine and examined using a Nikon Microphot-SA microscope and Optem 5X SC50 image capture system.

The detached tiller method used for okadaic acid (OA) uptake was carried out as described above, with the following modifications. Detached tillers were incubated for $24 \mathrm{~h}$ in the dark and in $100 \mathrm{mM}$ sucrose solution containing dilutions of a $10 \mathrm{mM}$ OA (Sigma) stock solution to give a concentration range from $100 \mathrm{nM}$ to $1 \mu \mathrm{M}$. Each treatment was repeated twice to nine times in separate experiments. Analysis of variance (ANOVA) was carried out using generalised linear models in Genstat 11th Edition.

\section{Results and discussion}

We have assessed whether it is possible to induce a $P h 1$ mutant pairing phenotype by delivering antisense oligodeoxynucleotides to silence these $c d k$-like genes at the onset of meiosis via a detached tiller incubation approach as described previously by Sun et al. 2005. Such an analysis would also confirm the involvement of these $c d k$-like genes in meiosis. The sense and antisense ODNs used were designed against the conserved regions of the Phl cdk-like genes, thus targeting all copies of the genes. Four 18 nucleotide-long antisense ODNs (and their corresponding sense copies) covered the start codon (ATG), a highly conserved region and the putative cyclin binding (DARTLRE) region of the $c d k$-like genes (Supplementary Fig. 1).

Meiosis was compared in immature anthers collected from sense and antisense ODN-treated detached wheat tillers. In sense ODN-treated tillers, first and second divisions of meiosis appeared normal. Meiocytes within each locule were all synchronised at the same stage of meiosis, and metaphase I showed normal pairing. However, in some anthers, young pollen cells appeared to contain micronuclei and a few binucleate young pollen cells were also seen. In antisense ODN-treated detached tillers, the meiocytes were asynchronous in more than $70 \%$ of the locules (Supplementary Fig. 2). The abnormalities were most pronounced during late stages of the second division. These included tetrads with missing nuclei and presence of micronuclei in the microspores (Supplementary Fig. 2). Chromosome pairing at metaphase I appeared normal except for the presence of univalents amongst the paired ring bivalents. This phenotype is similar to those meiotic abnormalities reported when disrupting the function of CDKA, the $c d c 2 / c d c 28$ homologue in Arabidopsis (Dissmeyer et al. 2007). Although the antisense ODN approach did not result in a phenotype that mimics that of the $P h 1$ deletion mutant, these observations did suggest that the $P h 1$ $c d k$-like genes play an important role in meiosis.

Expression studies revealed that deletion of the $P h 1 \mathrm{cdk}$ like genes on the $5 \mathrm{~B}$ chromosome results in increased transcription from the $c d k$-like genes on the $5 \mathrm{~A}$ and $5 \mathrm{D}$ chromosomes (Al-Kaff et al. 2008), suggesting that $c d k$ activity is increased rather than decreased by the absence of Ph1. Increased $c d k$ activity can be associated with premature chromosome condensation (Furuno et al. 1999). At the onset of meiosis, as the telomeres cluster into a bouquet, axial elements are generated by the condensation of sister chromatids onto a meiotic specific protein core that in plants contains ASY1, a homologue of Hop1p in yeast (Hollingsworth and Ponte 1997). 3-D immunofluorescence images of meiocytes labelled with anti-ASY1 revealed that in wheat-rye interspecific hybrids, chromosomes were less condensed when $P h 1$ was present than when it was absent both at the late telomere bouquet stage and when the bouquet disperses (Fig. 1; Supplementary Fig. 3). At both stages there were significant differences $(P<0.05)$ in the lengths of the labelled axial elements in the presence and absence of $P h 1$. Thus, in the absence of $P h 1$ in wheat interspecific hybrids, chromosomes condense earlier and chromosome pairing is induced between the related chromosomes. We suggest these two observations are linked and are consistent with the effect of $P h 1$ absence being an over-expression phenotype. It implies that the 

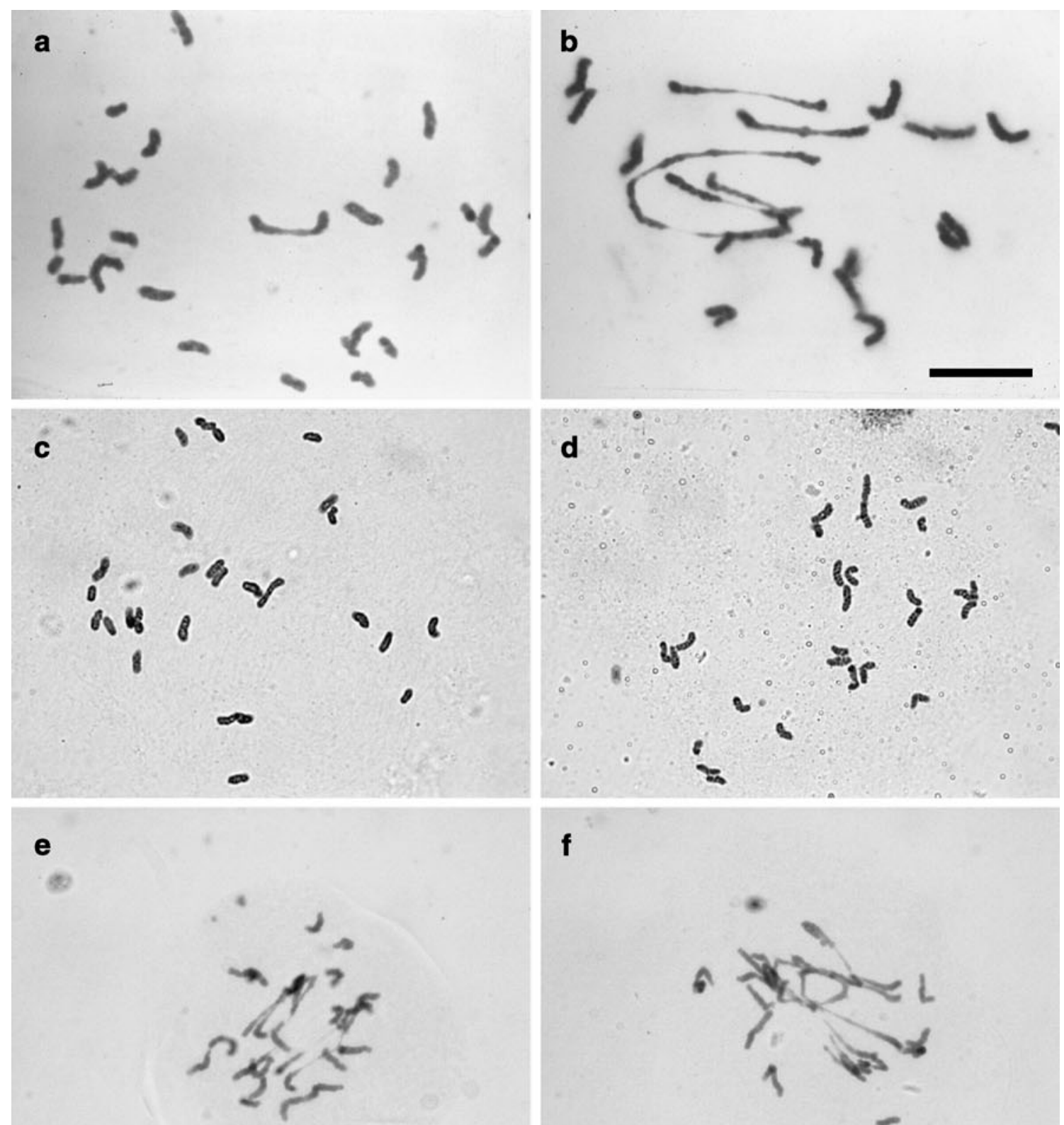

g
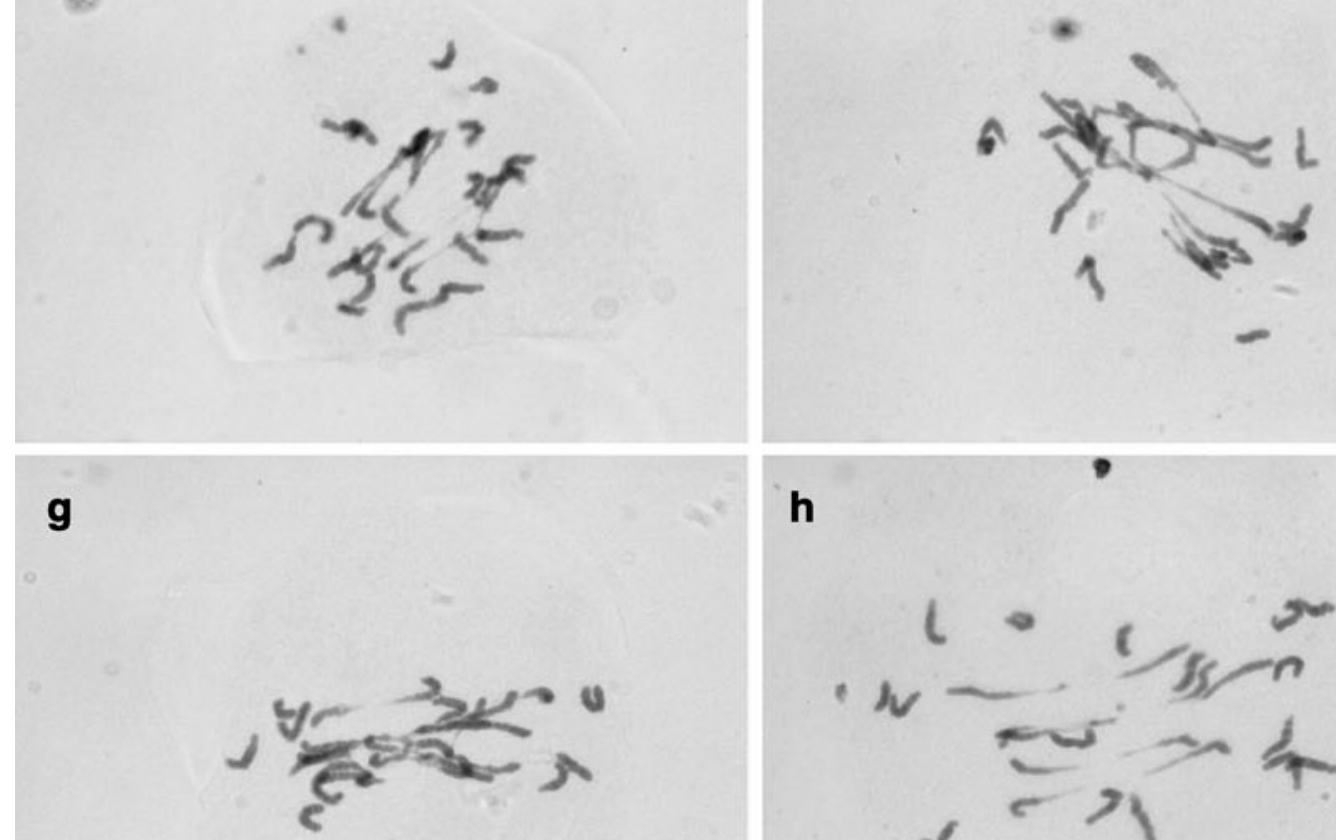

h

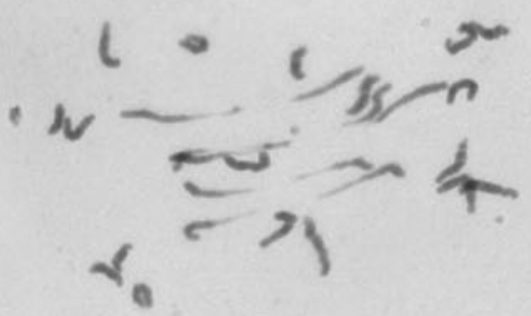


4Fig. 2 Induction of chromosome pairing in wheat-rye hybrids. Metaphase I chromosome spread from the hybrid with Phl (a) and without Phl (b). Metaphase I chromosome spread from detached tillers treated with sucrose $(\mathbf{c}, \mathbf{d})$ and with $100 \mathrm{nM} \mathrm{OA}(\mathbf{e}, \mathbf{f}, \mathbf{g}, \mathbf{h})$. Scale bar $10 \mu \mathrm{m}$

timing of chromosome condensation may be important in controlling the pairing of related chromosomes. Is it possible to induce early chromosome condensation during meiosis and hence phenocopy the Phl effect? CDKs phosphorylate proteins on serine and threonine amino acid residues and are involved in cell cycle regulation. In the absence of Ph1, cdk-like genes are over-expressed and there is an effect on chromosome condensation at early stages of meiosis. The phosphorylation targets for the Phl cdk-like proteins are most likely to be chromatin components that would allow these chromosome dynamic changes. Okadaic acid can be used to simulate the effects of the CDKs. OA is a potent protein serine/threonine phosphatase inhibitor which activates CDKs and induces premature chromosome condensation (Yamashita et al. 1990). We therefore investigated whether OA treatment could induce chromosome pairing in these hybrids even in the presence of $P h 1$.

Detached tillers of wheat-rye interspecific hybrid plants, which contain a haploid number of wheat and rye chromosomes (28 in total) were incubated in a sucrose solution with and without OA. Analysis of chromosome spreads at metaphase I revealed that OA affected chromosome pairing in a dose-dependent manner. There were very few chromosome arm associations observed in the sucrose control with most chromosomes visualised as univalents. However, as the OA concentration was increased from $100 \mathrm{nM}$ to $1 \mu \mathrm{M}$, the number of chromosome associations also increased, with the mean number of univalents per cell decreasing from 10 down to four for treatments with $1 \mu \mathrm{M}$ $\mathrm{OA}$ and the mean number of ungrouped chromosomes (a group being defined as three or more associated chromosomes) also decreasing from 15.3 at $100 \mathrm{nM}$ to 6.9 with $1 \mu \mathrm{M}$ OA (Supplementary Fig. 4). ANOVA revealed that the effect of $\mathrm{OA}$ on the number of univalents and ungrouped chromosomes per cell was highly significant $(P<0.001)$. However this analysis also revealed that treatments with concentrations greater than $200 \mathrm{nM}$ OA produce few rod bivalents suggesting little potential for genetic exchange. In contrast, treatment with $100 \mathrm{nM}$ OA resulted in a less marked chromosome association, but more importantly a strong increase in the number of rod bivalents (Fig. 2). Rod bivalent pairing is induced by timing the $100 \mathrm{nM}$ treatment to a period within $12 \mathrm{~h}$ of the onset of meiosis during pre-meiotic $S$ phase. However, this pairing is not observed in every treated anther within the spike, which suggests that the window for effective treatment is quite narrow. The chromosome pairing and associated multivalent formations observed at metaphase I after $100 \mathrm{nM}$ treatment were similar to the chromosome pairing behaviour observed when $P h l$ is deleted in the wheat-rye interspecific hybrid (Fig. 2). In 30 metaphase I chromosome spreads, an average of four chromosome arm associations per cell were observed following $100 \mathrm{nM} \mathrm{OA}$ treatment compared with an average of less than one arm association per cell following sucrose treatment. The $P h 1$ mutant has an average of seven arm associations per cell at metaphase I (Fig. 2; Dhalinal et al. 1977). This indicated that with the correct concentration of $\mathrm{OA}$, chromosome pairing can be induced in wheat-rye interspecific hybrid plants even in the presence of the Phl locus, mimicking the pairing observed in the absence of $P h 1$. Thus this approach of treating detached wheat tillers with a drug can in principal provide a powerful method to enhance genetic exchange between chromosomes.

Acknowledgements This work was supported by the UK Biotechnology and Biological Sciences Research Council. The authors would like to thank Dr Jason Able (University of Adelaide) who kindly provided the TaAsy1 antibody.

Open Access This article is distributed under the terms of the Creative Commons Attribution Noncommercial License which permits any noncommercial use, distribution, and reproduction in any medium, provided the original author(s) and source are credited.

\section{References}

Able J, Langridge P (2006) Wild sex in the grasses. Trends Plant Sci $11: 261-263$

Al-Kaff N, Knight E, Bertin I, Foote T, Hart N, Griffiths S, Moore G (2008) Detailed dissection of the chromosomal region containing the Ph1 locus in wheat Triticum aestivum: with deletion mutants and expression profiling. Ann Bot 101:863-872. doi: doi: $10.1093 / \mathrm{aob} / \mathrm{mcm} 252$

Boden SA, Langridge P, Spangenberg G, Able JA (2009) TaASY1 promotes homologous chromosome interactions and is affected by deletion of Phl. Plant J 57:487-497

Dhalinal HS, Gill BS, Waines JG (1977) Analysis of induced homoeologous pairing in a $p h$ mutant wheatxrye hybrid. J Hered 68:206-209

Dissmeyer N, Nowack MK, Pusch S, Stals H, Inze D, Grini PE, Schnittger A (2007) The cyclin-dependent kinase inhibitor KRP2 controls the onset of the endoreduplication cycle during Arabidopsis leaf development through inhibition of mitotic CDKA;1 kinase complexes. Plant Cell 19:972-985

Furuno N, den Elzen N, Pines J (1999) Human cyclin a is required for mitosis until mid prophase. J Cell Biol 147:295-306

Griffiths S, Sharp R, Foote TN, Bertin I, Wanous M, Reader S, Colas I, Moore G (2006) Molecular characterisation of $P h l$ as a major chromosome pairing locus in polyploid wheat. Nature 439:749 752

Hollingsworth NC, Ponte L (1997) Genetic interactions between HOP1, RED1 and MEK1 suggest that MEK1 regulates assembly of axial element components during meiosis in the yeast Saccharomyces cerevisiae. Genetics 147:33-42 
Prieto P, Moore G, Shaw P (2007) Fluorescence in situ hybridisation on vibratome sections of plant tissues. Nat Protoc 2:1831-1838

Riley R, Chapman V, Kimber G (1959) Genetic control of chromosome pairing in intergenic hybrids in wheat. Nature 183:1244-1246

Sanchez-Moran E, Benavente E, Orellana J (2001) Analysis of karyotypic stability of homoeologous-pairing $(p h)$ mutants in allopolyploid wheat. Chromosoma 110:371-377

Sun C, Hoglund AS, Olsson H, Mangelsen E, Jansson C (2005) Antisense oligodeoxynucleotide inhibition as a potent strategy in plant biology: identification of SUSIBA2 as a transcriptional activator in plant sugar signalling. Plant J 44:128-138

Viera A, Rufas JS, Martinez I, Barbero JL, Ortega S, Suj JA (2009) $\mathrm{CDK} 2$ is required for proper homologous pairing, recombination and sex-body formation during male mouse meiosis. J Cell Sci 122:2149-2159

Yamashita K, Yasuda H, Pines J, Ohtsuho M, Hunter T, Sugmura T, Nishimoto T (1990) Okadaic acid, a potent inhibitor of type 1 and type $2 \mathrm{~A}$ protein phosphatases, activates cdc2/H1 kinase and transiently induces a premature mitosis-like state in BHK21 cells. EMBO J 9:4331-4338

Yousafzai F, Al-Kaff N, Moore G (2010a) The molecular features of chromosome pairing at meiosis: the polyploidy challenge using wheat as a reference. Funct Integr Genomics 10:147-156

Yousafzai F, Al-Kaff N, Moore G (2010b) Structural and functional relationship between the $P h 1$ locus protein 5B2 in wheat and CDK2 in mammals. Funct Integr Genomics 10:157-166 